Tropical Journal of Pharmaceutical Research December 2010; 9 (6): 549-555

(C) Pharmacotherapy Group,

Faculty of Pharmacy, University of Benin

Benin City, 300001 Nigeria.

All rights reserved.

Research Article

Available online at http://www.tjpr.org

\title{
Comparative Costs of Antibacterial Usage in Sexually Transmitted Infections in a Nigerian Teaching Hospital
}

\author{
Ismail A Suleiman ${ }^{1^{\star}}$ and Fola Tayo ${ }^{2}$ \\ ${ }^{1}$ Department of Clinical Pharmacy and Pharmacy Practice, Niger Delta University, Wilberforce Island, PMB 071, \\ Yenagoa, Bayelsa State, ${ }^{2}$ Department of Clinical Pharmacy and Biopharmacy, Faculty of Pharmacy, University of \\ Lagos, College of Medicine Campus, PMB 12003, Lagos, Nigeria.
}

\begin{abstract}
Purpose: To evaluate the cost of antibacterial usage to patients in a tertiary health facility in Nigeria. Methods: Drug utilization evaluation was carried out retrospectively among patients with sexually transmitted infections (STIs) over a one-year period between 2005 and 2006 in Lagos University Teaching Hospital (LUTH), Nigeria. Case notes numbering 230 and containing 317 prescriptions were sampled consecutively with the aid of diagnostic coding cards. Relevant data including case demographics, diagnosis, prescribed drugs, and dosages were extracted and the associated costs analysed.

Results: A majority of the patients (82.3\%) were youths aged 21 - 40 years. Higher prevalence was observed among male patients (70.2 \%). The proportion of drugs prescribed in generic and branded names were 82.1 and $17.9 \%$, respectively. Total number of antibacterial doses was 7284 at a total cost of NGN239,420.00 (US\$1,841.69). Average antibacterial cost per patient was NGN1,040.96 (US\$8.00). Quinolones were the most widely prescribed class of antibacterial agents (31.1\% of cases) at a cost of NGN81,646.00 (US\$628.05) representing 34.1\% of the total antibacterial cost to patients.

Conclusion: This study indicates that antibacterial cost was high, relative to the purchasing power of the local populace, a large proportion of whom live below the poverty line (US\$1.00 per day). Furthermore, a greater proportion of the antibacterial cost was due to a few agents, the use of which should be evidence-based and closely monitored.
\end{abstract}

Keywords: Antibacterial therapy, Cost, Antibacterial utilization, Infectious diseases. 


\section{INTRODUCTION}

Sexually transmitted infections (STIs) such as syphilis, gonorrhoea, chancroid, chlamydia and non gonococcal urethritis are very common worldwide and a total prevalence of $21.5 \%$ has been reported in Nigeria [1]. STIs have considerable impact on the health of adolescents and young adults. Most of them are usually students, unemployed and stand the risk of poor management if contracted. In the year 2000, an estimated nine million cases of STIs occurred among persons aged 15 - 24 years in the United States [2]. Bowei, in 2006, estimated 332 million cases of STIs worldwide among adults aged 15 - 49 years [3]. A total prevalence of about 3.7 million cases was reported in Nigeria in 2001 [4]. It probably has not changed much since then. Therefore, STIs are of immense public health importance and is an area where more information is needed. STIs are also closely associated with HIV infection [5]. Relevant educational interventions in STIs management should enhance the capacity to reduce HIV transmission.

Expenditures on health are increasing worldwide and often health care systems have difficulties in meeting their obligations. Increasing longevity, higher prevalent rates of diseases, increasing antimicrobial resistance, more expensive diagnostic and therapeutic options, amongst others, often necessitate general increment in healthcare expenditures.

Allocated budget to health has been low in Nigeria $(<5 \%)$, which is considerably less than the $15 \%$ recommended by World Health Organisation (WHO) [6,7]. Consequently, healthcare managers in low-income countries, such as Nigeria, are indirectly being asked to do more with less and are, therefore, faced with challenge of rational decision-making [8]. Out of pocket expense, a major funding option of healthcare services in Nigeria is very limited, as many live below the poverty line, earning less than US\$ 1.00 per day [9].
Meanwhile, cost-related studies are very rare in many developing countries. In 1997, Taylor et al reported high cost of parenteral antibacterial therapy and long duration of hospitalization in Lagos, Nigeria [10]. In the report, a cost ranging from NGN600.00 (US\$4.62) to NGN32,987.00 (US\$253.75) was incurred by patients who were admitted for a period ranging from of 1 to 67 days. A gross irrational use of antibacterial agents was also documented with the use of 26 different antibacterial agents in the treatment of six major infections with a combination of two to five drugs per treatment regimen [9]. The cost implication of STIs treatment is compounded because of externality, resistance to antibacterial agents, complications such as infertility from pelvic inflammatory disease and non-adherence to treatment regimen.

This paper seeks to evaluate the cost to patients of antibacterial usage in a tertiary health facility in Lagos, Nigeria, as the findings could form the basis for decisionmaking and policy formulation.

\section{METHODS}

\section{Setting}

The study was carried out in the 764-bed Lagos University Teaching Hospital (LUTH), Lagos, Nigeria which is a tertiary health care centre. The hospital comprises Accident and Emergency Unit as well as various in-patient and general out-patient units, among others. Total patient turnover in the hospital is approx. 10,000 monthly. Various specialties and wards as well as diagnostic facilities are in place in the hospital. Antibacterial agents, if indicated, are prescribed in all the departments for surgical and medical cases. Each of the major hospital units has a pharmacy unit attached.

\section{Study design}

It was a retrospective drug utilization evaluation involving the use of consecutively 
sampled case notes over a one-year period between 2005 and 2006.

\section{Data collection}

Two hundred and thirty case notes containing 317 prescriptions pertaining to STIs were used. Relevant data such as date of visit, demographics, diagnosis, type of diagnostic test, prescribed drugs, dosages, frequency of dosing, and duration of therapy, were extracted. Follow-up visit, if any, and test of cure (a repeat diagnostic test within a month of therapy to ascertain complete eradication of infection or otherwise) were also collected using an appropriately designed and validated data collection form.

\section{Outcome measures}

Clinical and economic outcome variables were used. The clinical outcome measure used was assumed bacterial eradication. Economic outcome used was the antibacterial cost as a component of direct medical cost. There was no adjustment for discounting and inflation as all the costs occurred within one year of analysis. However, the exchange rate (between the Nigerian naira or NGN and United States dollar or US\$) at the time of analysis was used for consistency.

\section{Data analysis}

The collected data were analysed using Epi Info 2002 (CDC, USA). Mean cost per defined daily dose (C/DDD) [11] and the total cost of antibacterial agents per visit for each patient were computed for each drug. Also determined were the proportions of prescriptions in generic and branded names, the cost of each class of antibacterial agents, and number of visits. The volume of usage in doses for each drug and class of antibacterial agents were also computed. Data were analysed using Chi square test and Fisher's exact test (for small numbers) while mean costs were compared using Student's t-test. Descriptive statistics (mean and \%) were used in the presentation of results. At $95 \%$ confidence interval, a 2-tailed $p$-value less than 0.05 was considered significant.

\section{RESULTS}

\section{Demographic data and hospital visits}

A majority of the patients $(82.3 \%)$ were youths aged 21 - 40 years. First-time visit to the hospital by patients was $72.6 \%$ while follow-up visit to the hospital was very low $(27.4 \%)$, as shown in Table 1.

\section{Clinical variables}

Non-specific urethritis was the most prevalent STI and occurred among $43.2 \%$ of the patients, followed by vaginitis and syphilis/genital ulcer at the rate of 13.2 and $11.0 \%$, respectively. Others were genital warts $(3.8 \%)$, gonorrhoea (2.7\%), chlamydia $(1.6 \%)$, chancroid $(3.5 \%)$, pelvic inflammatory disease $(2.7 \%)$, cervicitis $(2.2 \%)$ and prostatitis $(2.2 \%)$.

Treatments were, in several cases, largely empirical as diagnostic tests were carried out in $53.6 \%$ of the patients, out of which $46.0 \%$ was microscopy culture and sensitivity (mcs). The test of cure rate for mcs test was very low (11.9\%), as Table 2 indicates. The most commonly isolated organism $(n=59)$ was Staphylococcus aureus, 20 (33.8\%). Others include Escherichia coli, 10 (16.9\%); Klebsiella spp, 8 (13.5\%); and other Staphylococus spp, 8 (13.5\%).

\section{Indicator variables}

The proportion of drugs prescribed in their generic names was $82.1 \%$ which is significantly higher than those prescribed in their brand names $\left(x^{2} ; p<0.05\right)$. Mean number of drugs per prescription was 1.8 and the volume of antibacterial agents prescribed in doses was 7284. Quinolones constituted $31.1 \%$ of the total prescriptions with ciprofloxacin being the most frequent (19.9 $\%)$ and $11.2 \%$ for other quinolones. 
Table 1: Demographic data and hospital visit among STIs patients

\begin{tabular}{llllll}
\hline $\begin{array}{l}\text { Age } \\
\text { (years) }\end{array}$ & \multicolumn{2}{c}{ Sex } & \multicolumn{2}{c}{ Visit } & $\begin{array}{l}\text { Total } \\
\text { n (\%) }\end{array}$ \\
\cline { 2 - 5 } & $\begin{array}{l}\text { Male } \\
\mathbf{n}(\%)\end{array}$ & $\begin{array}{l}\text { Female } \\
\mathbf{n}(\%)\end{array}$ & $\begin{array}{l}\text { First } \\
\mathbf{n}(\%)\end{array}$ & $\begin{array}{l}\text { Follow-up } \\
\text { n (\%) }\end{array}$ & \\
\hline $11-20$ & $11(3.5)$ & $16(5.1)$ & $21(6.6)$ & $6(1.9)$ & $27(8.5)$ \\
$21-30$ & $86(27.1)$ & $56(17.7)$ & $97(30.6)$ & $45(14.2)$ & $142(44.8)$ \\
$31-40$ & $103(32.5)$ & $16(5.1)$ & $89(28.1)$ & $30(9.5)$ & $119(37.5)$ \\
$41-50$ & $17(5.4)$ & $4(1.3)$ & $17(5.4)$ & $4(1.3)$ & $21(6.6)$ \\
$51-60$ & $4(1.3)$ & $0(0.0)$ & $3(0.9)$ & $1(0.3)$ & $4(1.3)$ \\
$>60$ & $3(0.9)$ & $1(0.3)$ & $3(0.9)$ & $1(0.3)$ & $4(1.3)$ \\
Total & $224(70.7)$ & $93(29.3)$ & $230(72.6)$ & $87(27.4)$ & $317(100.0)$ \\
\hline
\end{tabular}

STIs = sexually transmitted Infections

Table 2: Documented cases of diagnostic test among STI patients

\begin{tabular}{llll}
\hline Diagnostic test & $\begin{array}{l}\text { No. of samples } \\
\text { tested } \\
\mathbf{n}=\mathbf{3 1 7}(\%)\end{array}$ & $\begin{array}{l}\text { Positive result } \\
\mathbf{n}=\mathbf{1 7 0}(\%)\end{array}$ & $\begin{array}{l}\text { Test of } \\
\text { cure } \\
\mathbf{n}=59(\%)\end{array}$ \\
\hline Microscopy/Culture/Sensitivity & $112(35.3)$ & $59(46.0)$ & $7(11.9)$ \\
(VDT) & $54(17.0)$ & $6(11.1)$ & $0(0.0)$ \\
Chlamydial Test & $2(0.6)$ & $1(50.0)$ & $0(0.0)$ \\
TPHA & $2(0.6)$ & $0(0.0)$ & - \\
Total & $\mathbf{1 7 0 ( 5 3 . 6 )}$ & $\mathbf{6 6}(\mathbf{2 0 . 8})$ & $\mathbf{7 ( 1 1 . 9 )}$
\end{tabular}

STIs = Sexually transmitted infections; VDT = venereal disease test; TPHA = Treponema pallidum haemagglutination assay

\section{Cost variables}

The total antibacterial cost to all the patients over one year period was NGN239,420.00 (US\$1841.69). Of this, the quinolones formed the largest proportion - NGN81,646.00 (US\$628.95) or $34.1 \%$. Ciprofloxacin, which was the most prescribed quinolone (19.9\%), constituted $8.5 \%$ of the total antibacterial cost and $25.2 \%$ of the total quinolone cost while other quinolones with a prescription frequency of $11.2 \%$ constituted a statistically significant higher cost proportion of $74.8 \%$ of all the quinolones. A tenth $(11.1 \%)$ of the total antibacterial cost was accounted for by the cephalosporins but was prescribed at a rate of only $1.4 \%$. Azithromycin (a macrlide) also constituted $3.6 \%$ of total antibacterial cost despite being prescribed at a rate of 0.5 $\%$. The mean cost per single dose was NGN32.86 (US\$0.25) while the mean antibacterial cost per patient was
NGN1,040.00 (US\$8.00) as shown in Table 3.

\section{DISCUSSION}

The antibacterial drug cost (to the patients and health care systems) of treating the various infections was enormous. One thousand cases of urethritis, the most prevalent, and chlamydial infections required NGN664,000 (US\$5107.69) and NGN3.4 million (US\$26153.85), respectively. A median STIs treatment cost of US $\$ 17.80$ (NGN2314.00) has been reported in a systematic review for low-and middle-income countries [12]. These costs are huge and were borne mostly by youths who are economically disadvantaged being predominantly students or unemployed. This excludes other costs such as diagnostic test, transportation, consultation and loss of productivity. 
Table 3: Antibacterial cost for the treatment of sexually transmitted infections

\begin{tabular}{llccc}
\hline Drug class & \multicolumn{1}{c}{ Drug } & $\begin{array}{c}\text { Prescription } \\
\text { frequency } \\
\mathbf{n}(\%)\end{array}$ & $\begin{array}{c}\text { Total drug cost } \\
\text { NGN (US\$) }\end{array}$ & Drug cost (\%) \\
\hline Quinolones & Ciprofloxacin & $113(19.9)$ & $20,556.00(158.12)$ & 8.5 \\
& Others & $64(11.2)$ & $61,090.00(469.92)$ & 25.6 \\
Tetracycline & Subtotal & $177(31.1)$ & $81,646.00(628.95)$ & 34.1 \\
& Doxycycline & $163(28.6)$ & $33,018.00(253.98)$ & 13.8 \\
& Tetracycline & $3(0.5)$ & $640.00(4.92)$ & 0.3 \\
Cephalosporin & Subtotal & $166(29.2)$ & $33,658.00(258.90)$ & 14.0 \\
& Ceftriaxone & $3(0.5)$ & $14,218.00(109.37)$ & 6.0 \\
Macrolides & Cefuroxime & $5(0.9)$ & $12,300.00(94.62)$ & 5.1 \\
& Subtotal & $8(1.4)$ & $26,518.00(203.98)$ & 11.1 \\
& Erythromycin & $65(11.4)$ & $33,616.00(258.58)$ & 14.0 \\
& Azithromycin & $3(0.5)$ & $8606.00(66.2)$ & 3.6 \\
Others & Subtotal & $68(11.9)$ & $42,222.00(324.78)$ & 17.5 \\
& Subtotal & $\mathbf{1 5 0 ( 2 6 . 3 )}$ & $\mathbf{5 5 , 3 7 6 . 0 0 ( 4 2 5 . 9 7 )}$ & $\mathbf{2 3 . 3}$ \\
& Grand total & $\mathbf{5 6 9 ( 1 0 0 . 0 )}$ & $\mathbf{2 3 9 , 4 2 0 . 0 0 ( 1 8 4 1 . 6 9 )}$ & $\mathbf{1 0 0 . 0}$ \\
\hline
\end{tabular}

Note: Exchange rate at the time of the study was US\$1 = NGN130; no adjustment for inflation or discounting was made

Accurate diagnosis is important to ensure optimal use of limited resources but this is not affordable to most of the affected patients and the health care system, hence syndromic management of STIs has been recommended [4]. Antibacterial therapy needs to be specific while empiric prescription should be minimized by strengthening necessary capacity building such as improving diagnostic facilities. This, in turn, will reduce cost and improve the quality of management. In this study, generic prescribing was more prevalent than brand name prescription; this should be sustained as it also reduces costs significantly [13].

The documented cases of diagnostic tests was found in about half of the patients, which implies that treatments were, in a large number of cases, empirical, and this could be a major factor in the development of bacterial resistance $[14,15]$. Non-affordability of these tests could, however, be another problem encountered by the patients.

The much lower cost of ciprofloxacin relative to the total quinolone antibacterial cost, despite its higher prescribing rate, is an indication that other quinolones should be restricted to situations in which ciprofloxacin is not indicated. Combination of ciprofloxacin, doxycycline and metronidazole, which was widely used in this study in syndromic management of STIs, have previously been evaluated [16]. Low rate of MCS tests, poor follow-up, and very low test of cure in this study make it difficult to ascertain the actual degree of cure achieved. Moodley et al [16] examined the use of a syndromic algorithm in rural South Africa among 616 women. The women were treated with ciprofloxacin 500 $\mathrm{mg}$ in one dose, doxycycline $200 \mathrm{mg}$ daily for 7 days, metronidazole tablet $2 \mathrm{~g}$ in a single dose, and metronidazole $2 \mathrm{~g}$ daily for 5 days for women with lower abdominal pain. The cure rates for chlamydia, gonorrhoea and trichonomas ranged from 78 to $90 \%$. However, only $52 \%$ of women with vaginosis responded to therapy.

In the present study, other frequently prescribed drugs include erythromycin and metronidazole and this was at a much lower cost to patients than the quilolones. These agents are affordable to most patients but should be used rationally. Misuse should be 
avoided to prevent or minimise resistance development, thereby reducing costs of antibacterial therapy. The drug distribution system in Nigeria needs to be improved upon and over the counter sales of antibacterial agents need to be vigorously checked. Regulatory institutions should be strengthened to achieve this in order to minimize bacterial resistance to these lifesaving antibacterial agents that are rapidly losing value. Cephalosporins, which were prescribed at a very low rate, constituted a relatively high cost of antibacterial therapy. This implies their usage has to be closely monitored from an economic perspective to minimize the huge burden of total antibacterial cost to the health care system.

The national cost implication of antibacterial agents for the reported STIs prevalence of 3.7 million in Nigeria [4] is about NGN3.85 billion (US\$29.63 million) or more. Part of this huge sum could be channeled to other areas or diseases if incidence of STIs is reduced and treatments are more cost-effective.

\section{CONCLUSION}

Antibacterial cost to patients and the nation at large is enormous and this calls for prudent use of these agents. In addition, a greater proportion of the antibacterial cost is accounted for by few high-cost agents, the use of which should be evidence-based and closely monitored.

\section{ACKNOWLEDGEMENT}

The cooperation of the hospital management, physicians, pharmacists, medical record officers, nurses of Lagos University Teaching Hospital, Nigeria, among others, is appreciated.

\section{REFERENCES}

1. Otunoye O. Prevalence of Sexually Transmitted Diseases in Nigeria. West Afr J of Med 2002; 2 (2): 152-156.
2. Weinstock $H$, Berman S, Cates W Jr. Sexually transmitted diseases among American Youths: incidence and prevalence estimates. Perspectives on Sexual and Reproductive Health 2004; 36(1): 6-10.

3. Bowie WR. Drug Therapies for sexually transmitted diseases: clinical and economic considerations. Sexually Transmitted Infections 2006; 82: 201; doi:10.1136/ sti.2005.018960

4. Federal Ministry of Health (FMOH) Syndromic Management of Sexually Transmitted Infections. A Manual for Health Workers. Abuja, Nigeria; 2001

5. World Health Organization (WHO). Preventing HIVIAIDS in Young People: A Systematic Review of the Evidence from Developing Countries, WHO Technical Report Series no. 938, Geneva: WHO, 2006.

6. Federal Ministry of Health (Nigeria). Financing and sustainability of Tuberculosis Control in Nigeria. Ministerial Conference on TB \& Sustainable Development Amsterdam, 22-24 Mar 2000.

7. Department of International Development (DFID) Report on Nigeria, 2006.

8. World Health Organization. Identification of needs in Health Economics in Developing Countries. WHO/TFHE/95.3. 1995

9. United Nations Development Programme (UNDP). Technical Report On Nigeria, 2006.

10. Taylor O, Chukwani CM, Eniojukan JF, Fajemirokun O. Socioeconomic evaluation of parenteral antibacterial therapy in Lagos University Teaching Hospital. Results of a retrospective survey. West Afr J Pharm 1997; 11(2): 78-86

11. Neithimer $A$ I. The defined daily dose system (DDD) for drug utilization review. Hosp Pharm; 1986; 21: 233-241.

12. Terris-Prestholt $F$, Vyas $S$, Kumaranayake $L$, Mayaud $P$, Watts $C$. The cost of treating curable sexually transimtitted infections in lowand middle income countries: a systematic review. Sex Transm Dis. 2006 Oct; 33(10 Suppl): S153-166.

13. World Health Organizations. Health Economics, Drug and Health Sector reform. WHO Task force on Health Economics; 1996.

14. Okeke IN, Lamikanra A, Edelman R. Socioecnomic and behavioral factors leading to acquired bacterial resistance to antibiotics in developing countries. Emerg Inf Dis 1999; 5: 1

15. Albrich WC, Monnet DL, Harbarth S. Antibiotic selection pressure and resistance in streptococcus pneumoniae and Streptococcus pyogenes. Emerg Inf Dis 2004; 10: 514-517.

16. Moodley $P$, Wilkinson D, Connoly $C$, Sturm AW. Syndromic management of female genital discharge does not curb ineffectiveness in rural African population. Program and abstract of the $40^{\text {th }}$ Interscience Conference on Antimicrobial Agents and Chemotherapy; 
Suleiman \& Tayo

Toronto, Ontario. Canada; September 17-20,

2000. Abstact 1194. 